\title{
Opioid use among veterans undergoing major joint surgery managed by a multidisciplinary transitional pain service
}

\author{
Michael J Buys (10 1,2 Kimberlee Bayless, ${ }^{2}$ Jennifer Romesser, ${ }^{3}$ Zachary Anderson, ${ }^{2}$ \\ Shardool Patel, ${ }_{1}^{2}$ Chong Zhang, ${ }_{1}^{4}$ Angela P Presson, ${ }^{4}$ Benjamin S Brooke ${ }^{5,6}$
}

${ }^{1}$ Department of Anesthesiology, University of Utah, Salt Lake City, Utah, USA

${ }^{2}$ Anesthesiology, VA Salt Lake City Health Care System, Salt Lake City, Utah, USA

${ }^{3}$ Psychology, VA Salt Lake City Health Care System Mental Health Services, Salt Lake City, Utah, USA

${ }^{4}$ Internal MedicineEpidemiology, University of Utah Health Sciences Center, Salt Lake City, Utah, USA

${ }^{5}$ Surgery, University of Utah School of Medicine, Salt Lake City, Utah, USA

${ }^{6}$ Surgery, VA Salt Lake City Health Care System, Salt Lake City, Utah, USA

\section{Correspondence to}

Dr Michael J Buys, Department of Anesthesiology, University of Utah, Park City, UT 84098, USA; michael.buys@hsc.utah.edu

Received 9 June 2020 Revised 20 July 2020 Accepted 25 July 2020 Published Online First 26 August 2020

\section{ABSTRACT}

Background Chronic postsurgical pain and opioid use is a problem among patients undergoing many types of surgical procedures. A multidisciplinary approach to perioperative pain management known as a transitional pain service (TPS) may lower these risks.

Methods This retrospective cohort study was conducted at the Salt Lake City VA Medical Center to compare patients undergoing elective primary or revision total knee, hip, or shoulder replacement or rotator cuff repair in the year before (2017) and after (2018) implementation of a TPS. The primary outcome is the proportion of patients taking opioids 90 days after surgery. Secondary outcomes include new chronic opioid use (COU) after surgery as well as the proportion of previous chronic opioid users who stopped or decreased opioid use after surgery.

Results At 90 days after surgery, patients enrolled in TPS were significantly less likely to be taking opioids (13.4\% TPS vs $27.3 \%$ pre-TPS; $p=0.002$ ). This relationship remained statistically significant in a multivariable logistic regression analysis, where the TPS group had 69\% lower odds of postoperative COU compared with the preintervention group (OR: 0.31; 95\% Cl: 0.14 to 0.66; $p=0.03$ ). Opioid-naive patients enrolled in TPS were less likely to have new COU after surgery $(0.7 \%$ TPS vs $8.4 \%$ pre-TPS; $p=0.004)$. Further, patients enrolled in TPS with existing COU prior to surgery were more likely to reduce or completely stop opioid use after surgery (67.5\% TPS vs $45.3 \%$ pre-TPS; $p=0.037)$ as compared with pre-TPS.

Conclusions These data suggest that a TPS is an effective strategy for preventing new COU and reducing overall opioid use following orthopedic joint procedures in a Veterans Affairs hospital.

\section{INTRODUCTION}

Despite many advances over the past decade, perioperative pain management strategies continue to be inadequate for a high percentage of surgical patients. Ranges from $10 \%$ to $49 \%$ have been reported for patients experiencing chronic postsurgical pain (CPSP), ${ }^{1-5}$ which is defined generally as pain in the surgical area that lasts beyond the expected time frame of surgical healing. Analgesic therapies are often only marginally effective, and/or have significant deleterious effects associated with them. Consequently, as many as $14 \%$ of previously opioid-naive patients go on to develop chronic opioid use (COU) after surgery. ${ }^{6-13}$

Historically, perioperative pain management has fallen primarily under the direction of the surgical team whereby pain management is discussed briefly with the patient, but typically without any standardized education or coordination with primary care providers after surgery. When patients have ongoing surgical pain beyond the normal follow-up period, primary care and emergency room providers are frequently tasked with deciding whether to continue opioids and other pain medications. Together, these factors can lead to fragmentation of perioperative pain management and inappropriate continuation of opioid medications.

One solution to address fragmentation of perioperative pain management is to deliver opioid education, risk modification, cognitive behavioral therapy, and prescribing recommendations by a single transitional pain service (TPS). This type of multidisciplinary pain approach has been previously described, although other TPS programs have not been designed to engage surgical patients beginning in the preoperative period. ${ }^{14-16}$ In 2018, we designed and implemented a novel TPS at the Salt Lake City VA Medical Center that engages at-risk patients starting in the preoperative period and follows them until up to 6 months after surgery. The goals of this TPS are to (1) reduce pain suffering through education, risk modification, cognitive interventions, and optimization of pharmacologic and non-pharmacologic therapy; (2) eliminate the development of new COU after surgery; and (3) assist prior chronic opioid users to decrease or completely taper off opioid use after surgery.

This study was designed to evaluate whether implementation of a TPS would reduce opioid use among veterans undergoing major joint surgery, a patient population at high risk for CPSP. ${ }^{17}$ We hypothesized that a multidisciplinary approach to pain management would decrease fragmentation during care transitions and significantly reduce opioid use after orthopedic surgery for both chronic opioid users and opioid-naive patients.

\section{METHODS}

\section{TPS intervention}

The TPS intervention consists of comprehensive pain management and care coordination delivered by a multidisciplinary team. This team is made up of anesthesiologists with specialization in acute pain 
management, nurse practitioners with experience in both acute and chronic pain management, nurse care coordinators, and a psychologist. These multidisciplinary providers work together as an integrated team to deliver comprehensive pain management for any surgical patient at risk for CPSP and COU. Patients are eligible for TPS engagement based on presence of individual risk factors such as a history of substance use disorder or COU, or if they are undergoing a surgical procedure such as total joint replacement associated with a high-risk for CPSP. ${ }^{17}$ TPS engagement of these patients occurs during all three main phases of a surgical episode of care: (1) the preoperative period, (2) the surgical hospitalization, and (3) postoperative recovery period up to 6 months.

\section{Preoperative period}

Patients are identified for enrollment in TPS at the time they are scheduled for elective surgical procedures. A TPS nurse contacts each patient before surgery and performs a directed history including mental health diagnoses, pain history, opioid use history, and baseline patient-reported pain measures using Patient Reported Outcome Measurement Information System (PROMIS) instruments as well as pain catastrophizing scale scores. ${ }^{18}$ Patients receive preoperative education either through a 1 hour surgical expectations class or one-on-one visit, where topics such as pain after surgery, analgesic strategies, appropriate use of opioids, and non-pharmacologic pain coping strategies are discussed. Individual sessions with a psychologist are also offered prior to surgery with a focus on Mindfulness-Based Intervention (MBI), and Acceptance and Commitment Therapy (ACT). All patients are then reviewed by the anesthesiologist and nurse practitioner prior to surgery to develop individualized plans for perioperative pain management. This may include a discussion with the patient regarding preoperative opioid taper, opioid-free surgery and recovery, buprenorphine strategy, or other multimodal analgesic techniques such as peripheral nerve blocks. Any changes for opioid taper or buprenorphine use are discussed directly and done in coordination with the prescribing provider.

\section{Surgical hospitalization period}

Individualized pain management plans and Enhanced Recovery After Surgery protocols are implemented including preoperative non-steroidal anti-inflammatory drug (NSAID), acetaminophen, and gabapentinoid, local infiltrative anesthesia by the surgeon, and regional anesthesia, where appropriate. Postoperative scheduled acetaminophen, NSAID, and gabapentinoid were provided with breakthrough opioids as needed for postoperative analgesia. Daily rounds are conducted on hospitalized patients by the multidisciplinary TPS team and any new pain management recommendations are made to the surgical team to include discharge medications and opioid taper plan. During their in-hospital stay, patients are offered individual sessions with a psychologist on a daily basis or more frequently for mindfulness meditation and/or relaxation techniques.

\section{Postoperative period}

Follow-up telephone calls are made by the TPS nurse at postdischarge days $2,7,10,14,21,28$, and at least monthly until 6 months postoperative. During each call, 24-hour morphine equivalent daily dose (MEDD), PROMIS scores, date of last opioid use, and number of opioid tabs remaining after date of last opioid use are recorded. Outpatient sessions with the psychologist are offered with a continuation of ACT Matrix and MBI used as well as assessment and referral for appropriate long-term mental health services. The anesthesiologist and nurse practitioner provide opioid taper support and prescribe alternative analgesic therapies when needed. Any change in medications is done through close communication and coordination with the surgical team, primary care, mental health and/or chronic pain providers. For patients with chronic pain and $\mathrm{COU}$, transition to a chronic pain team is offered and facilitated.

\section{TPS implementation}

The TPS intervention as described above was implemented at the VA Salt Lake City Medical Center in January 2018 as a 1 year pilot. During this pilot period, we enrolled all patients scheduled to undergo primary or revision total knee, hip, or shoulder surgery or rotator cuff surgery based on the established risk of CPSP and opioid use among this patient population. After a year, the TPS program was expanded to include other surgical patient populations. To support implementation, electronic decision support tools were created, including electronic medical record order sets to standardize practice, and decision-support dashboards to facilitate case review at the patient-level by all members of the TPS team.

\section{Study design}

We used a pre-post study design to compare changes in postoperative opioid use among patients undergoing orthopedic surgery procedures before and after implementation of the TPS intervention. Patients in the pre-TPS group who had primary or revision total knee, hip, or shoulder surgery or rotator cuff surgery at the Salt Lake City VAMC between January and December 2017 were compared with patients undergoing the same surgical procedures who were enrolled in TPS during 2018. Veterans who had a second surgery or that passed away within 90 days after their joint surgery were excluded from this analysis. This study was approved by the University of Utah Institutional Review Board and VA Research Review Committee, and informed consent was waived.

\section{TPS and pre-TPS groups}

For the pre-TPS group, medical records and state-controlled substance databases were reviewed to ascertain baseline demographics, history of substance use disorder, depression, anxiety, post traumatic stress disorder (PTSD) or other mental health diagnosis, and use of opioid medications before and after surgery. MEDD was calculated at baseline prior to surgery and at 90 days after discharge based off of active prescriptions in the medical record and/or state-controlled substance database at those time points. Number of opioid tablets prescribed at discharge was recorded. Patients were designated as chronic opioid users if they had received a prescription of opioids for either 60 continuous days prior to surgery, or if they filled three or more prescriptions for opioids within 180 days prior to surgery. Veterans who did not meet these criteria were considered opioid naive. New COU after surgery was defined as having either an active opioid prescription at 90 days postdischarge or filling an opioid prescription between 90 and 120 days after discharge.

Patients in the TPS cohort had opioid use history and MEDD documented in TPS clinic notes using patient report as well as medical record and controlled substance database verification prior to surgery and at 90 days after discharge. Number of opioid tablets prescribed at discharge was recorded. In addition, history of mental health disorders and substance abuse history were recorded at baseline prior to surgery. 


\section{Outcome measures}

The primary outcome measure for this study was the proportion of patients taking any opioid medication at 90 days after surgery. Secondary outcomes included the proportion of COU patients who were either weaned off opioids or decreased their opioid dose as compared with preoperative levels, the proportion of non-opioid use (NOU) patients still on opioids after 90 days, and the number of opioid tablets prescribed at discharge.

\section{Statistical analysis}

Baseline patient characteristics were summarized stratified by the pre-TPS and post-TPS exposure groups. Continuous variables were summarized as mean, SD, median, IQR, and range; and analyzed using a t-test for approximately normally distributed variables and a Wilcoxon rank-sum test otherwise. Categorical variables were summarized as count (\%) and analyzed using a $\chi^{2}$ test. A Cochran-Armitage trend test was used to compare 90-day outcomes coded as an ordinal variable (off opioids, reduced, same, increased) among the patients in pre-TPS and post-TPS who had COU. We also used a $\chi^{2}$ test to compare off or reduced versus same or increased opioid use. We first compared predictor variables between the pre-TPS and post-TPS NOU and COU groups using univariate analysis. A multivariable logistic regression model was then used to assess the relationship between TPS exposure status and postoperative COU, adjusting for age, type of procedure, number of days in hospital, and COU at baseline. Covariates were selected for adjustment in logistic regression models if they met statistical significance at the $p<0.1$ level in univariate analyses. ORs were reported with 95\% CIs and $\mathrm{p}$ values. Statistical significance was assessed at the 0.05 level, and all tests were two-sided. Preimplementation and postimplementation outcomes for number of opioid tablets prescribed at discharge and percentage of new COU are presented in a Statistical Process Control Chart. ${ }^{19}$ All analyses were conducted using R V.3.6.1 (R Foundation for Statistical Computing, Vienna, Austria).

\section{RESULTS}

A total of 164 patients met criteria for the TPS program between January and December 2018, and were compared with 172 patients in pre-TPS from the year prior that underwent the same types of orthopedic procedures. This cohort included 53 (31\%) patients in pre-TPS and $37(23 \%)$ patients in the TPS cohort with a history of COU before surgery. The characteristics of patients in pre-TPS and TPS groups prior to surgery are shown in table 1.

After implementation of TPS there was a significant reduction in the number of opioid tablets prescribed to patients at the time of hospital discharge (TPS: $45(25,60)$ vs pre-TPS: $80(60,90)$; $\mathrm{p}<0.001$; figure 1). Opioid tablets prescribed by month are shown in figure 2. This finding was consistent for patients who were opioid naive as well as on chronic opioid therapy before surgery.

The decrease in opioid use associated with the TPS intervention was found to extend beyond 3 months after surgery for patients who were either opioid naive or on chronic opioids before surgery. Only 22 (13.4\%) patients under TPS were still on opioids after 90 days as compared with 47 (27.3\%) patients in pre-TPS $(p=0.002)$. Among individuals who were opioid naive prior to surgery, only a single patient who received the TPS intervention continued opioids beyond 90 days after hospital discharge $(0.7 \%$ vs $8.4 \% ; \mathrm{p}=0.004)$ as compared with 10 patients from the pre-TPS period. Figure 3 shows this trend over time. Similarly, the TPS intervention was effective for patients
Table 1 Characteristics of patients enrolled in the TPS and patients in no TPS undergoing the same major joint procedures during the year prior

\begin{tabular}{|c|c|c|c|}
\hline & No TPS & TPS & \\
\hline Variables & $\mathrm{n}=172$ & $n=164$ & $P$ value \\
\hline Age, mean (SD) & $65.7(9.9)$ & $64.2(12.0)$ & 0.21 \\
\hline Sex, male, $n(\%)$ & $159(92.4)$ & $145(88.4)$ & 0.21 \\
\hline BMI, median (IQR) & $31.0(28-35)$ & $31.6(29-35)$ & 0.39 \\
\hline History of mental health disorders, $\mathrm{n}(\%)$ & $81(47.1)$ & $79(48.2)$ & 0.84 \\
\hline History of substance use disorders, $\mathrm{n}(\%)$ & $25(14.5)$ & $22(13.4)$ & 0.77 \\
\hline Surgical procedure, n (\%) & & & 0.38 \\
\hline Rotator cuff repair & $40(23.3)$ & $33(20.1)$ & \\
\hline Total hip arthroplasty & $46(26.7)$ & $34(20.7)$ & \\
\hline Total knee arthroplasty & $66(38.4)$ & $72(43.9)$ & \\
\hline Total shoulder arthroplasty & $20(11.6)$ & $25(15.2)$ & \\
\hline Hospital length of stay, median (IQR) & $2.0(1-2)$ & $2.0(1-2)$ & 0.58 \\
\hline \multicolumn{4}{|l|}{ Opioid use history } \\
\hline COU, n (\%) & $53(30.8)$ & $37(22.6)$ & 0.09 \\
\hline Preop MEDD, mean (SD) & $15.4(35.8)$ & $10.3(31.2)$ & 0.07 \\
\hline
\end{tabular}

BMI, body mass index; COU, chronic opioid use; MEDD, morphine equivalent daily dose; TPS, transitional pain service.

on chronic opioids before surgery. As shown in table 2, among chronic opioid users there was a significant increase in the proportion of patients enrolled in the TPS program who either completely stopped their opioids or decreased their preoperative opioid dose $(67.5 \%$ vs $45.3 \%)$ when compared with pre-TPS $(\mathrm{p}=0.037)$.

As a result, fewer chronic opioid users engaged by TPS were found to have increased their opioid dose at 90 days (5.5\% TPS vs $15.1 \%$-Pre-TPS) following surgery. However, the trend across these ordered levels was not statistically significant $(p=0.054)$.

Multivariable logistic regression analysis showed 69\% lower odds of postoperative COU in the TPS group compared with

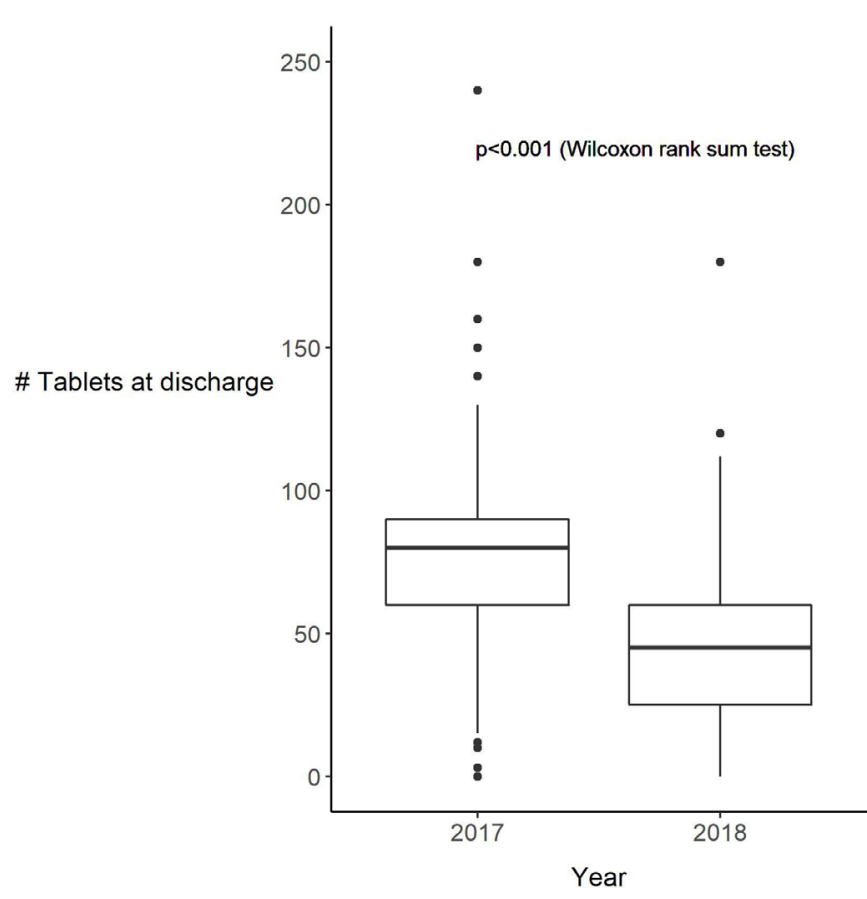

Figure 1 Number of opioid tablets prescribed at hospital discharge before and after implementation of a TPS. TPS, transitional pain service. 


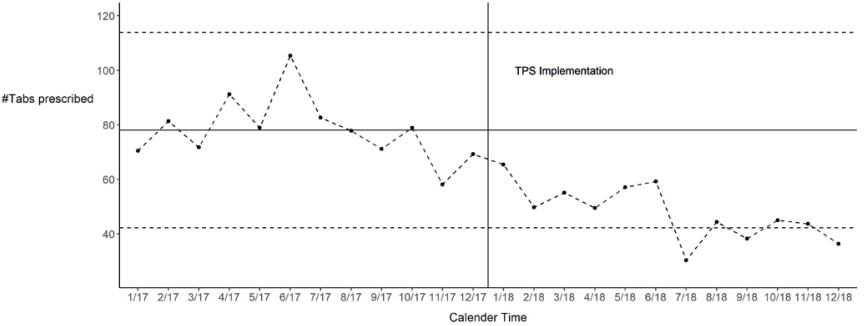

Figure 2 Statistical control chart for number of opioid tablets prescribed at hospital discharge, by month, before and after implementation of a TPS. Dots are average number of tabs prescribed at discharge per month. Solid horizontal line is the mean number of tabs prescribed at discharge in 2017. Two dashed horizontal lines represent 3SDs from the mean of the monthly number of tabs at discharge for year 2017. In 2018, all months had an average \#tabs at discharge below the central line (2017 average), 3 months (7, 9, and 12) were outside of the lower boundary (mean -3SD), suggesting special causes of variation. TPS, transitional pain service.

the pre-TPS group (OR: $0.31 ; 95 \% \mathrm{CI}: 0.14$ to $0.66 ; \mathrm{p}=0.03$; table 3).

COU at baseline was associated with over 40 times higher odds of postop COU (OR: 43.2; 95\% CI: 20.1 to 102.1; p<0.001). In this model, patient age, type of procedure, and hospital days were not independently associated with COU after surgery adjusting for TPS intervention and baseline COU.

\section{DISCUSSION}

Perioperative care coordination for pain management is commonly fragmented and increases the risk for COU after surgery. Implementing a TPS has been suggested as one approach to address this issue and to optimize the perioperative pain management strategy. ${ }^{20-22}$ Here we describe the successful implementation of a TPS in a Veterans Affairs (VA) medical

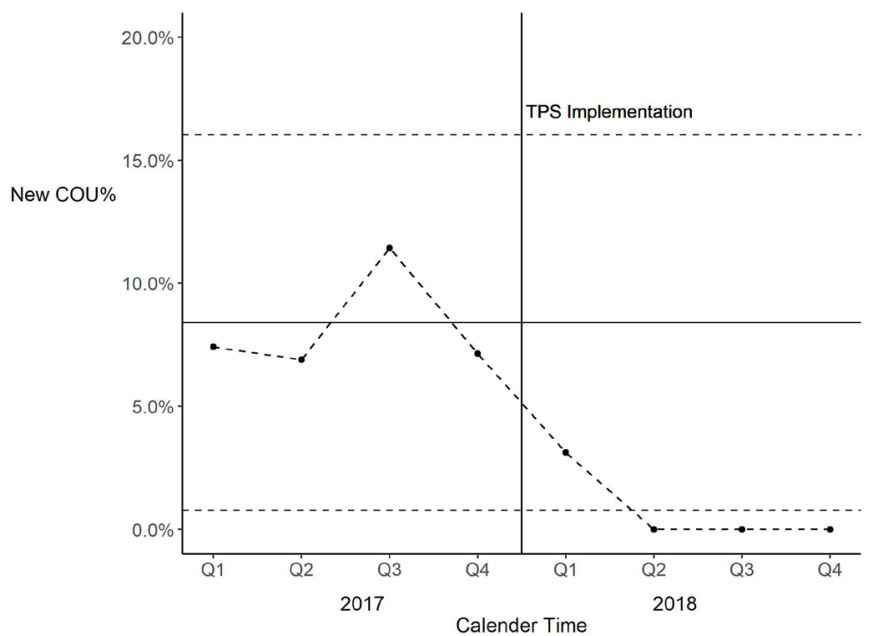

Figure 3 Statistical control chart for proportion of opioidnaive veterans with new persistent opioid use before and after implementation of a TPS. Dots are \% new COU in each quarter. Solid horizontal line is the mean new COU\% in 2017. Two dashed horizontal lines are 3SD from the mean new COU\% in 2017. In 2018, new COU\% in all four quarters were below the central line, quarters 2-4 had new COU\% outside of the lower boundary (mean -3SD), suggesting special causes of variation. COU, chronic opioid use; TPS, transitional pain service.
Table 2 90-Day outcomes among patients with history of COU who received the TPS intervention as compared with standard care during prior year (no TPS)

\begin{tabular}{lrrrr}
\hline & No TPS & & \multicolumn{1}{c}{ TPS } & \\
\cline { 2 - 2 } Outcome & $\mathrm{n}=53$ & & $\mathrm{n}=37$ & P value \\
\hline Off or reduced opioid use, $\mathrm{n}(\%)$ & $24(45.3)$ & & $25(67.5)$ & \multirow{2}{*}{$0.037^{*}$} \\
Off all opioids, $\mathrm{n}(\%)$ & $16(30.2)$ & & $16(43.2)$ & \\
Reduced opioid use, $\mathrm{n}(\%)$ & $8(15.1)$ & $9(24.3)$ & \\
Same or increased opioid use, $\mathrm{n}(\%)$ & $29(54.7)$ & & $12(32.5)$ & \\
Same opioid dose as baseline, $\mathrm{n}(\%)$ & $21(39.6)$ & & $10(27)$ & \\
Increased opioid use, $\mathrm{n}(\%)$ & $8(15.1)$ & $2(5.5)$ & \\
\hline
\end{tabular}

${ }^{*} \chi^{2}$ test comparing off or reduced versus same or increased.

COU, chronic opioid use; TPS, transitional pain service.

center that utilizes a multidisciplinary and multimodal approach across preoperative, surgical hospitalization, and postoperative transitions of care to address the problem of fragmented care coordination. Our data show that when patients receive preoperative education and are followed closely for at least 6 months after surgery, overall postoperative opioid use can be significantly reduced for both previously opioid naive as well as chronic opioid users. It is noteworthy that among opioid-naive patients in this study, TPS achieved substantial reduction of new COU after surgery. In addition, the majority of patients with prior COU were able to transition completely off opioids within 90 days after surgery or experience a reduction in their opioid dose. Together, these findings support the role for a TPS in optimizing perioperative pain management and reducing COU among at-risk patients.

Numerous evidence-based strategies have been promoted and/ or put into practice during the preoperative, intraoperative, and postoperative period as a means to reduce opioid use and prevent CPSP. This includes individually tailored programs for preoperative education or pain management planning ${ }^{23-25}$; use of multimodal analgesia including regional or neuraxial techniques or non-opioid systemic medications ${ }^{25}$; use of non-pharmacologic modalities such as cognitive-based intervention ${ }^{26}$; as well as coordinated approach to postdischarge instructions and transitions of care. ${ }^{26}$ Each of these individual strategies by themselves has been shown to have some effect on improving postoperative pain management. And all of these measures are supported by current professional guidelines for the management of postoperative pain. $^{27}$

There has been limited data, however, to show the beneficial effect when these therapeutic modalities are all bundled together into a single perioperative intervention. A multidisciplinary TPS program provides the mechanism within a health

Table 3 Multivariable regression analysis to assess independent factors associated with COU following orthopedic joint surgery

\begin{tabular}{llc}
\hline Variable & ORs $(95 \% \mathrm{Cl})$ & P value \\
\hline Post-TPS vs Pre-TPS & $0.32(0.14$ to 0.69$)$ & 0.005 \\
Age at discharge & $1.01(0.97$ to 1.05$)$ & 0.61 \\
Type of surgery & & \\
$\quad$ Hip arthroplasty & $0.86(0.24$ to 3.16$)$ & 0.82 \\
$\quad$ Total knee arthroplasty & $1.74(0.58$ to 5.77$)$ & 0.34 \\
\hline Total shoulder arthroplasty & $2.25(0.59$ to 8.78$)$ & 0.24 \\
Length of hospital stay & $1.08(0.82$ to 1.34$)$ & 0.52 \\
Preop MEDD>0 & $1.08(1.06$ to 1.11$)$ & $<0.001$ \\
\hline
\end{tabular}

MEDD, morphine milligram equivalent daily dose; TPS, transitional pain service. 
system for delivering multiple interventions to patients at high risk for CPSP. ${ }^{1420}$ Our study demonstrates the effectiveness of a TPS that integrates preoperative education and individual pain management planning in addition to postoperative care. This is a central component of our approach based on collective evidence showing that managing patient expectations before surgery reduces patient anxiety, perioperative opioid consumption, and hospital length of stay. ${ }^{28-30}$

The other main function of a TPS is to ensure that this personalized pain management plan is carried out across all transitions of care. A TPS coordinates care across all transitions in the inpatient and outpatient setting and effectively lowers the risk that patient information is miscommunicated among surgical and non-surgical providers. ${ }^{21}$ This type of service is well-suited to an integrated healthcare system such as the VA given the shared electronic medical record system, the ability to collect and track data in both inpatient and outpatient settings, and the relative ease of communication among surgeons, primary care, and mental health providers. Moreover, coordination of care is facilitated given that most veterans who have surgery at a VA hospital typically receive all of their care through the VA healthcare system. In fact, the electronic note templates we created were designed to be utilized at other VA hospitals during future TPS dissemination, allowing common data elements to be captured and helping to guide best practices for perioperative pain management. Further, the real-time capturing of data through patient care notes allowed us to develop a provider-facing clinical decision tool that facilitated regular follow-up at specified intervals and ease of visualizing pertinent patient information in a single place.

Given the resource-intensive nature of a TPS like ours, we recognize that there is a need to demonstrate scalability and cost-effectiveness. After the first year of implementation of our TPS (2018), we expanded to include veterans having all types of surgery and have enrolled $>1000$ additional veterans since that time. We are also currently performing a cost analysis study. Additional work is needed to evaluate if a model such as this would be successful and feasible at other institutions with different financial models and higher surgical volume.

Our study has several limitations to discuss. First, we used a pre-post study design that may have been confounded by temporal changes in opioid prescribing patterns and as well as regression to the mean. Second, data between time periods was not collected in the same manner. While data were collected prospectively among patients enrolled in the TPS cohort, we had to perform retrospective electronic chart abstraction among patients in the historic pre-TPS group. As such, some degree of ascertainment bias may have been introduced. Third, there may be some unmeasured confounders in our analysis that influence risk of COU between patients enrolled in the TPS and the historic pre-TPS group and contribute to treatment selection bias. Finally, the reduction in COU observed among patients in the TPS program may have been influenced by other VA initiatives designed to reduce opioid use implemented during the study time period.

\section{CONCLUSIONS}

The significant reduction in COU observed in this pilot study of patients who had orthopedic surgery underscores the potential for a multidisciplinary TPS approach to comprehensive perioperative pain management. Future directions include evaluating TPS among other surgical patient populations as well as exploring implementation of the service for veterans who receive care through the VA but choose to have surgery outside of the
VA medical system. In addition, it will be important to identify those who are at risk for prolonged opioid use and poor functional outcomes to better focus resources on those who would most benefit from a TPS.

Correction notice This article has been corrected since it published Online First. The author name, Benjamin Brooke, has been corrected.

Twitter Kimberlee Bayless @DrKimBaylessNP

Contributors MJB contributed to the conception, design, data collection, data analysis and interpretation, and manuscript preparation. KB contributed to the conception, design, data collection, data analysis and interpretation, and manuscript preparation. JR contributed to the conception, design, data collection, data analysis and interpretation, and manuscript preparation. ZA contributed to the conception, design, data collection, data analysis and interpretation, and manuscript preparation. SP contributed to the conception, design, data collection, data analysis and interpretation, and manuscript preparation. CZ contributed to the data analysis and interpretation, and manuscript preparation. APP contributed to the data analysis and interpretation, and manuscript preparation. BB contributed to the conception, design, data analysis and interpretation, and manuscript preparation.

Funding Funding for this study was received from the VA Whole Health Initiative, the VA Center of Innovation, the VA Office of Rural Health, and the National Institutes of Health Grant (UL1TR002538).

Competing interests None declared.

Patient consent for publication Not required.

Ethics approval University of Utah and Salt Lake City VA Institutional Review Board IRB_00106087.

Provenance and peer review Not commissioned; externally peer reviewed.

Data availability statement Data are available upon reasonable request. The data that support the findings of this study are available from the corresponding author (MJB), upon reasonable request.

\section{ORCID iD}

Michael J Buys http://orcid.org/0000-0001-8222-1461

\section{REFERENCES}

1 Ilfeld BM, Madison SJ, Suresh PJ, et al. Persistent postmastectomy pain and pain-related physical and emotional functioning with and without a continuous paravertebral nerve block: a prospective 1-year follow-up assessment of a randomized, triple-masked, placebo-controlled study. Ann Surg Oncol 2015;22:2017-25

2 Fletcher D, Stamer UM, Pogatzki-Zahn E, et al. Chronic postsurgical pain in Europe: an observational study. Eur J Anaesthesiol 2015;32:725-34.

3 Huang A, Azam A, Segal S, et al. Chronic postsurgical pain and persistent opioid use following surgery: the need for a transitional pain service. Pain Manag 2016:6:435-43.

4 Montes A, Roca G, Sabaté S, et al. Chronic post-surgical pain after herniorrhaphy, hysterectomy and thoracotomy: incidence of pain at 3 and 12 months. GENDOLCAT study. Eur J Anaesthesiol 2012;29:4.

5 Barrington JW, Lovald ST, Ong KL, et al. Postoperative pain after primary total knee arthroplasty: comparison of local injection analgesic cocktails and the role of demographic and surgical factors. J Arthroplasty 2016;31:288-92.

6 Harbaugh CM, Nalliah RP, Hu HM, et al. Persistent opioid use after wisdom tooth extraction. JAMA 2018;320:504-6.

7 Lennon MJ, Torlot FJ, Micallef J, et al. Persistent opioid use after arthroplasty. Anaesth Intensive Care 2018;46:629-30.

8 Namba RS, Inacio MCS, Pratt NL, et al. Persistent opioid use following total knee arthroplasty: a signal for close surveillance. J Arthroplasty 2018;33:331-6.

9 Johnson SP, Chung KC, Zhong L, et al. Risk of prolonged opioid use among Opioid-Naïve patients following common hand surgery procedures. J Hand Surg Am 2016:41:947-57

10 Schoenfeld AJ, Nwosu K, Jiang W, et al. Risk factors for prolonged opioid use following spine surgery, and the association with surgical intensity, among OpioidNaive patients. J Bone Joint Surg Am 2017;99:1247-52.

11 Brummett CM, Waljee JF, Goesling J, et al. New persistent opioid use after minor and major surgical procedures in US adults. JAMA Surg 2017;152:e170504.

12 Bartels K, Fernandez-Bustamante A, McWilliams SK, et al. Long-term opioid use after inpatient surgery - A retrospective cohort study. Drug Alcohol Depend 2018; 187:61-5.

13 Hansen CA, Inacio MCS, Pratt NL, et al. Chronic use of opioids before and after total knee arthroplasty: a retrospective cohort study. J Arthroplasty 2017;32:811-7. 


\section{Original research}

14 Katz J, Weinrib A, Fashler SR, et al. The Toronto General Hospital transitional pain service: development and implementation of a multidisciplinary program to prevent chronic postsurgical pain. J Pain Res 2015;8:695-702.

15 Walters TL, Mariano ER, Clark JD. Perioperative surgical home and the integral role of pain medicine. Pain Med 2015;16:1666-72.

16 Vetter TR, Kain ZN. Role of the perioperative surgical home in optimizing the perioperative use of opioids. Anesth Analg 2017;125:1653-7.

17 Pinto PR, McIntyre T, Ferrero R, et al. Risk factors for moderate and severe persistent pain in patients undergoing total knee and hip arthroplasty: a prospective predictive study. PLoS One 2013;8:e73917.

18 Sullivan MJL, Bishop SR, Pivik J. The pain Catastrophizing scale: development and validation. Psychol Assess 1995; 7:524-32.

19 Benneyan JC, Lloyd RC, Plsek PE. Statistical process control as a tool for research and healthcare improvement. Qual Saf Health Care 2003;12:458-64.

20 Glare P, Aubrey KR, Myles PS. Transition from acute to chronic pain after surgery. Lancet 2019;393:1537-46.

21 Clarke $\mathrm{H}$, Ladha K. Time for accountability and change: institutional gaps in pain care during the opioid crisis. Br J Anaesth 2019;122:e90-3.

22 Klueh MP, Hu HM, Howard RA, et al. Transitions of care for postoperative opioid prescribing in previously Opioid-Naïve patients in the USA: a retrospective review. $J$ Gen Intern Med 2018:33:1685-91.
23 Dunn LK, Sun EC. Mind over matter: reducing perioperative opioid use through patient education. Anesth Analg 2020;130:556-8.

24 Horn A, Kaneshiro K, Tsui BCH. Preemptive and preventive pain Psychoeducation and its potential application as a multimodal perioperative pain control option: a systematic review. Anesth Analg 2020;130:559-73.

25 Lee $\mathrm{BH}, \mathrm{Wu} \mathrm{CL}$. Educating patients regarding pain management and safe opioid use after surgery: a narrative review. Anesth Analg 2020;130:574-81.

26 Reynolds MAH. Postoperative pain management discharge teaching in a rural population. Pain Manag Nurs 2009;10:76-84.

27 Chou R, Gordon DB, de Leon-Casasola OA, et al. Management of postoperative pain: a clinical practice guideline from the American pain Society, the American Society of regional anesthesia and pain medicine, and the American Society of Anesthesiologists Committee on regional anesthesia, executive Committee, and administrative Council. J Pain 2016;17:131-57

28 Crowe J, Henderson J. Pre-arthroplasty rehabilitation is effective in reducing hospital stay. Can J Occup Ther 2003;70:88-96.

29 Rucinski K, Cook JL. Effects of preoperative opioid education on postoperative opioid use and pain management in orthopaedics: a systematic review. J Orthop 2020;20:154-9.

30 EGBERT LD, Battit GE, Welch CE, et al. Reduction of postoperative pain by ENCOURAGEMENT and instruction of patients. A study of doctor-patient RAPPORT. $N$ Engl J Med 1964;270:825-7. 